\title{
Surface-Coil MRI for Small Peripheral Choroidal Melanoma: Imaging in a Rabbit Eye Model
}

\author{
Larissa loannidi ${ }^{a}$ b Konstantinos Seliniotakis ${ }^{a, b}$ Georgios Bontzos ${ }^{a, b}$ \\ George Sourvinos $^{\mathrm{e}}$ Viktor Haniotis $^{\mathrm{d}}$ Irene Tsiapa $^{\mathrm{c}}$ Thomas G. Maris ${ }^{\mathrm{c}}$ \\ Efstathios T. Detorakis ${ }^{a, b}$

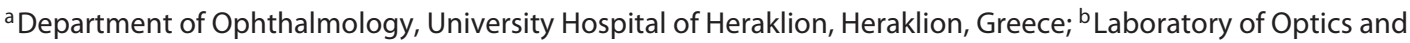 \\ Vision, University of Crete, Heraklion, Greece; ' Department of Medical Physics, University of Crete, Heraklion, Greece;

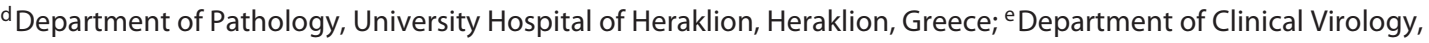 \\ University Hospital of Heraklion, Heraklion, Greece
}

\section{Keywords}

MRI - Melanoma · Choroid · Surface coils · Rabbits

\begin{abstract}
Introduction: Imaging of small peripheral choroidal melanomas remains challenging in clinical and experimental settings, requiring wide-field ophthalmoscopy modalities. In this study, we applied surface-coil MRI to assess imaging feasibility for small peripherally located choroidal melanoma in a rabbit eye model. Methods: A female albino rabbit with a body weight of $2.8 \mathrm{~kg}$ was immunosuppressed with intramuscular cyclosporine $15 \mathrm{mg} / \mathrm{kg}$ daily for 3 days prior to inoculation of 14 million 92.1 line melanoma cells into the peripheral suprachoroidal space of the right eye. The experimental animal continued to receive the same dosage of cyclosporine daily for 26 days postinoculation until the day of sacrifice. On that interval, it underwent surface-coil MRI scanning using various protocols and models subsequently sacrificed to allow histological evaluation. A comparative examination of various MRI sequences for optimal lesion imaging was performed, based on semiquantitative criteria. $\boldsymbol{R} \boldsymbol{e}$ -
\end{abstract}

\section{KARGER}

(c) 2018 S. Karger AG, Basel

E-Mail karger@karger.com

www.karger.com/oop sults: Imaging quality and superimposed artifacts varied across different sequences and the optimal tumor delineation from adjacent tissues was achieved in T1-weighted sequences. Conclusions: MRI of small peripheral choroidal melanomas is feasible in a rabbit model using surface coils, particularly in T1-weighted sequences. Findings may be used as a basis for further development of this technique for experimental and clinical applications.

(c) 2018 S. Karger AG, Basel

\section{Introduction}

Conventional fundus cameras produce $35-50^{\circ}$ photographs of the central fundus or composite fundus images (resulting from the combination of peripherally focused images), for peripheral lesions $[1,2]$. The latter, however, remain operator and patient dependent and may be difficult to produce and interpret [1]. Although imaging modalities for the peripheral fundus, such as wide-field scanning laser ophthalmoscopy, digital wide-field angiography, or wide-field fundus autofluorescence, have 
now emerged, they are still under technological development and not widely available [1]. The need for monitoring the development and progression of peripheral fundus lesions applies for nonneoplastic conditions, such as peripheral vascular conditions [3], diabetic retinopathy [3], or retinopathy of prematurity [4] but perhaps more importantly for neoplastic conditions, such as peripheral melanotic lesions and especially choroidal melanomas [5, 6]. Differential diagnosis and monitoring of progression is particularly important in choroidal melanoma, since it is a potentially sight-threatening or life-threatening condition and even modalities with peripheral imaging capabilities may not provide accurate information on critical tumor parameters, such as tumor height $[7,8]$. Importantly, A-scan and B-scan ultrasound, which is the typical diagnostic tools to assess tumor height in choroidal melanomas may produce suboptimal images for peripheral sites and small lesions [8]. Conventional head-coil MRI might not be adequate for smaller lesions $[9,10]$ but surface-coil MRI provides higher resolution and signal-tonoise ratio for the eyeball [11]. A previous study by Hosten et al. [12] has employed surface-coil MRI for choroidal melanoma imaging for the detection of potential extraocular extension of large choroidal melanomas with a sensitivity of $86 \%$ and a specificity of $71 \%$. In our case, we are focusing on creating a shorter-term animal model with regard to the retention period of the experimental animals, taking into account the smaller size of the developing volume. The challenge in this case is the difficulty of imaging and monitoring such a small volume with conventional techniques. The present study aims at evaluating the feasibility of surface-coil MRI for the imaging of small peripheral choroidal melanomas in a rabbit eye model.

\section{Methods}

This prospective experimental (animal) study was performed at the Department of Ophthalmology, the Laboratory of Optics \& Vision, and the Department of Medical Physics of the University of Crete. The protocol was approved by the ethics committee of our hospital and experiments were conducted according to the national (Greek) laws concerning animal experiments. In the context of attempting to develop an experimental choroidal melanoma model, different protocols were developed using different modes of immunosuppression and methods of implanting melanocytic cells. In this article, we describe the development of choroidal melanoma based on the work of Blanco et al. [13, 14].

A female New Zealand nonpigmented (albino) adult rabbit was used, with a body weight of $2.8 \mathrm{~kg}$. Frozen 92.1 cell line melanoma cells were used to produce an experimental choroidal melanoma model (Fig. 1b). The cells were obtained from the Labora- tory of Ophthalmology at Leiden University, The Netherlands (courtesy of Prof. Martine Jager and Dr. Mieke Versluis). The cells were received in frozen $\left(-80^{\circ} \mathrm{C}\right)$ vials, each containing 5-10 million cells in $20 \%$ of DMSO, and stored in liquid nitrogen. For defrosting and culturing the cells, the suspension was immersed in a $37^{\circ} \mathrm{C}$ water bath and once it was almost completely thawed is was transferred to $9 \mathrm{~mL}$ of Eagle's modified essential medium (Biosera, UK) and centrifuged to obtain a cell pellet. The cell pellet was washed once more with a volume of medium and seeded in a T25 culture flask. The culture medium was the RPMI 1640 (Gibco, ThermoFisher Scientific, Waltham, MA, USA), supplemented with $10 \%$ FBS, 3 mM L-glutamine, $2 \%$ penicillin/streptomycin. One or two weeks after thawing the cells, they were split twice a week on a 1:3 ratio.

The experimental animal was systematically immunosuppressed by intramuscular injections of $15 \mathrm{mg} / \mathrm{kg} /$ day of cyclosporin A (Sandimmune $50 \mathrm{mg} / \mathrm{mL}$, Novartis, NJ, USA), as previously described $[13,14]$, for 3 days prior to melanoma cell inoculation and continued to receive the same dosage of cyclosporin A for 26 days postinoculation, until the day of sacrifice. For both the inoculation surgery and the MRI scanning process, the experimental animal was anesthetized with intramuscular injections of a mixture containing ketamine hydrochloride $(50 \mathrm{mg} /$ $\mathrm{kg}$ ) and xylazine $(5 \mathrm{mg} / \mathrm{kg})$. Additional topical anesthesia to the eyes was applied (1 drop of sodium chloride proxymetacain, Alcaine, ALCON Lab, Hellas AEBE). The procedure for the inoculation of 14 million cells in the peripheral suprachoroidal spaces included a superior $180^{\circ}$ conjunctival peritomy at the corneoscleral limbus area, followed by the formation of a local superficial scleral flap. The cells were placed in a $1-\mathrm{mL}$ syringe with a 27-G needle, which was advanced under the superficial scleral flap into the underlying suprachoroidal space (Fig. 1a). The cells were then injected in a watertight fashion and the superficial scleral flap was sutured to prevent cell escape into the subconjunctival space. Postoperatively, it received eye drops of tobramycin $(0.3 \%)$-dexamethasone $(0.1 \%)$ (Tobradex, Alcon Laboratories, Inc., Fort Worth, TX, USA) until the time of sacrifice. During the experiment, the animal was examined every 5 days using a portable slit lamp for anterior segment and indirect ophthalmoscopy after pupil dilation (using eye drops of tropicamide $1 \%$, cyclopentolate $1 \%$, phenylephrine $2.5 \%$ ). Following examination, MRI was performed.

MR imaging was performed using a clinical 1.5-T whole-body superconducting imaging system (MAGNETOM Sonata/Vision, Siemens Healthcare, Erlangen, Germany), equipped with highperformance gradients (gradient strength: $45 \mathrm{mT} / \mathrm{m}$, slew rate: 200 $\mathrm{mT} / \mathrm{m} / \mathrm{ms}$ ) utilizing a standard circular polarized body coil as a transmitter and a linear polarized small circular surface coil (external diameter $=5 \mathrm{~cm}$, active internal diameter $=3 \mathrm{~cm}$ ) as a receiver. The small surface coil was mounted and immobilized on an in-house designed MR-compatible holder apparatus (Fig. 1c). Following appropriate anesthesia, as described above, the experimental animal was positioned on a purpose-designed container with one linear polarized small surface coil deployed over its right orbit (Fig. 1d). The final image voxel dimensions were $0.1 \times 0.1 \mathrm{~mm}^{2}$ in-plane spatial resolution and $0.6 \mathrm{~mm}$ cross-plane spatial resolution (slice thickness) as previously described $[15,16]$. Axial space filling sequences of $0.6 \mathrm{~mm}$ were obtained using a $256 \times 224$ matrix over a $5 \times 4.5 \mathrm{~cm}$ field of view. 
Fig. 1. a The surgical procedure consisted of general anesthesia and inoculation of cells in the peripheral suprachoroidal space. b Frozen 92.1 cell line melanoma cells were used for the inoculation surgery. c The animal is positioned in a specially designed container under a standard circular polarized body coil. d The small surface coil was deployed over the right orbit.
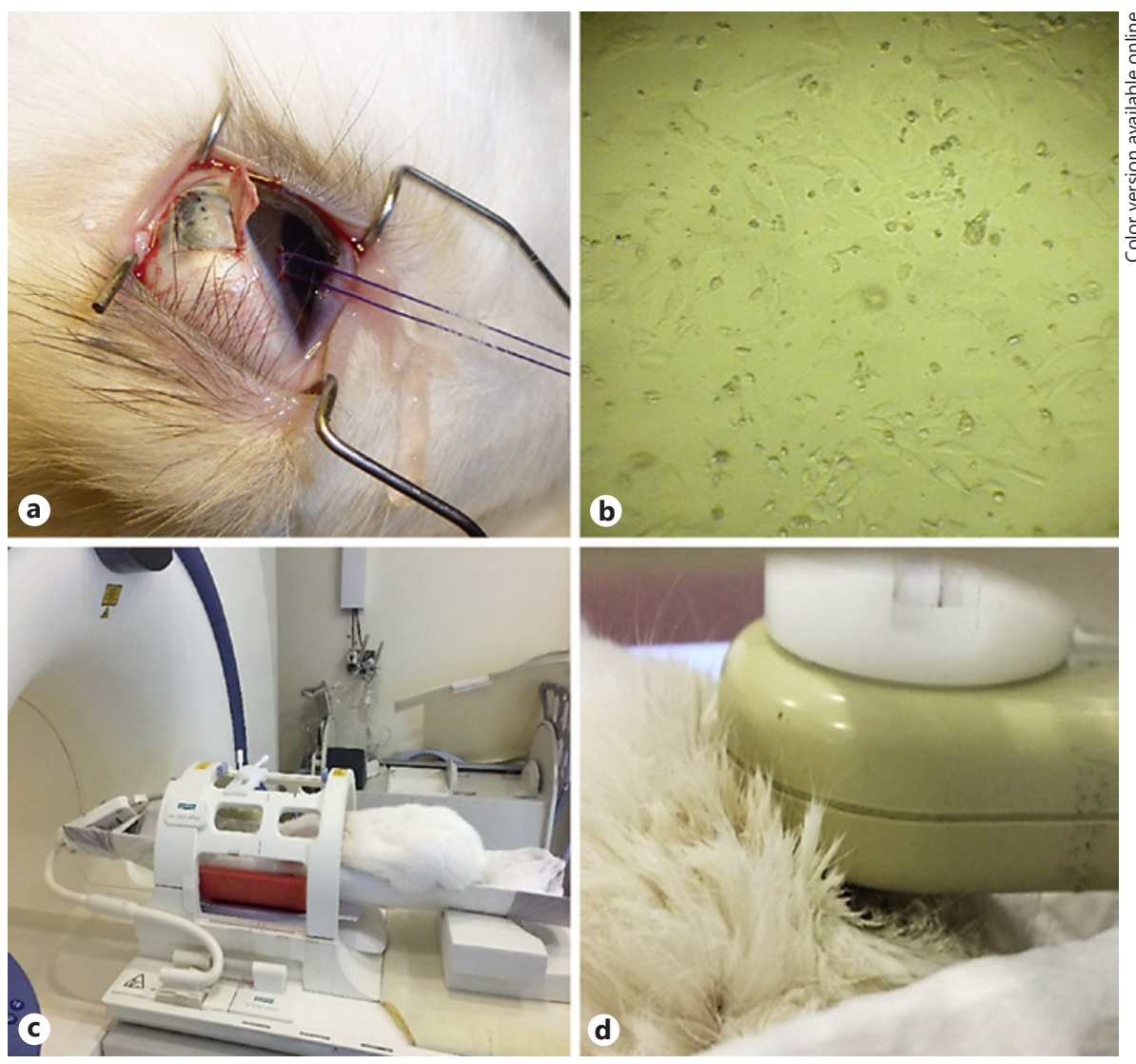

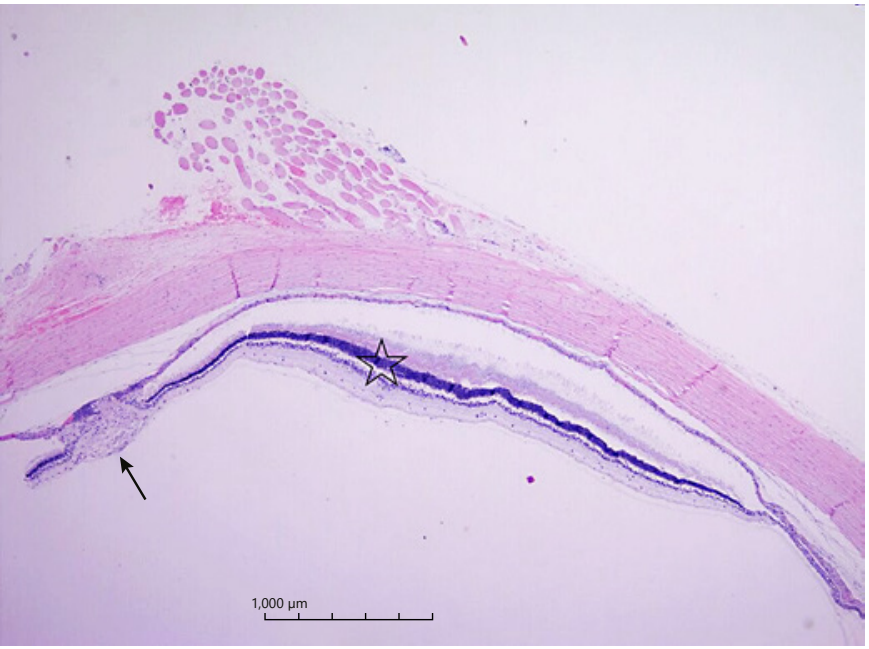

Fig. 2. Light microscopic evaluation of the enucleated eye with tumor tissue (arrow). The tumor was vascular and its cells were polygonal with vesicular cell nuclei containing prominent nucleoli. Numerous mitotic figures were noted in the microscopic field and a choroidal thickening of $391.09 \mu \mathrm{m}$ (star).
Table 1. Typical imaging parameters used for the sequences that were applied for MR eye examination

\begin{tabular}{lcc}
\hline & 3D-VIBE & 3D-CISS \\
\hline Slice thickness, mm & 0.6 & 0.6 \\
Fumber of slices & 30 & 30 \\
Field of view, mm & 60 & 60 \\
In-plane resolution, mm & 0.1 & 0.1 \\
TR, ms & 11.80 & 12.98 \\
TE, ms & 5.02 & 5.5 \\
Flip angle, degrees & 15 & 70 \\
Scan time per volume, min & 3.50 & 3.40 \\
\hline
\end{tabular}

The comprehensive HR-MR imaging protocol consisted of one 3D T1w sequence (3D-VIBE, Volume Interpolated Breath hold Examination) and one heavily T2-weighted sequence (3DCISS, Constructive Interference on the Steady State). The most important sequence parameters are shown in Table 1 . Both sequences were obtained in planes parallel to the equator of the eyes.

DICOM images were analyzed with the eFilm workstation (Merge Healthcare 900, Hartland, WI, USA) and the EvoRAd RISPACS workstation (EvoRAd Medical Information Systems, Her- 
Fig. 3. a T1-w sequence (3D-VIBE) of the model's eye illustrating the tumor area in the ciliary body at 12 o'clock position (arrow). b T2-w sequence (3D-VIBE) of the model's eye cannot delineate the tumor area from the surrounding healthy tissue. c Four ROIs obtained from the relative eye segments and one ROI obtained from the image background for the noise estimation. AC, anterior chamber; SCB, superior area of the ciliary body; ICB, inferior area of the ciliary body; $\mathrm{VH}$, vitreous humor; Bg, background.
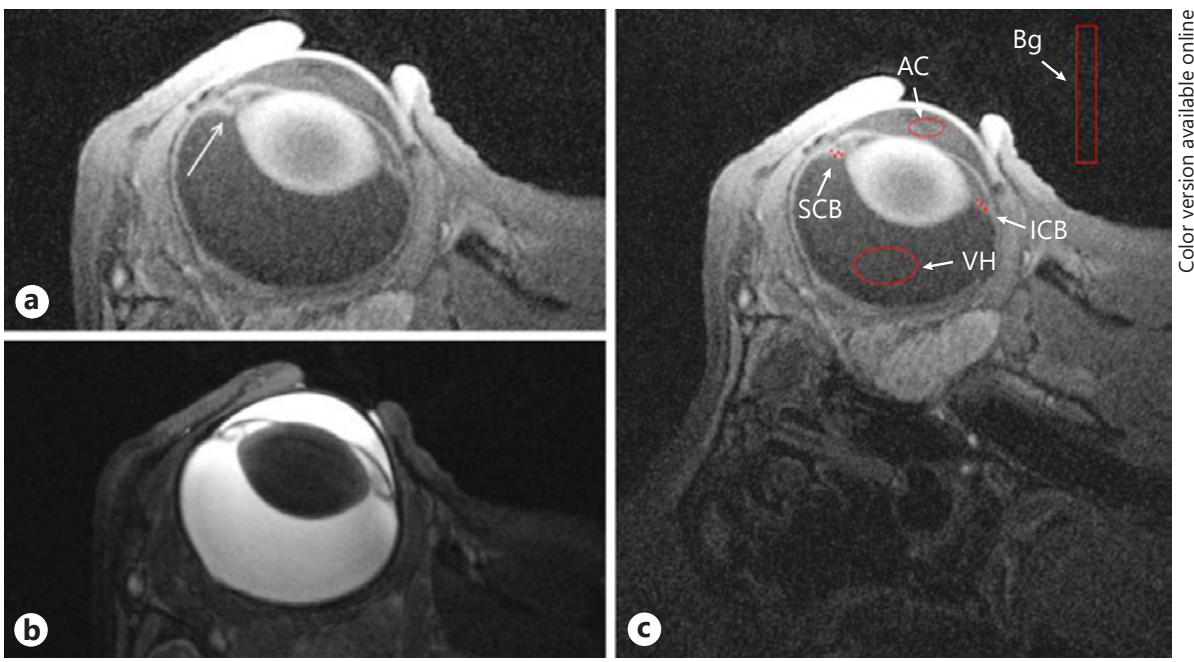

aklion, Crete, Greece). Analysis included the identification of the tumor within the region of interest (ROI), corresponding to the melanoma cell inoculation site at the superior peripheral fundus just behind the iris root.

The maximal diameter and surface area occupied by the tumor was manually delineated and measured in coronal, axial, and transverse sections using the incorporated measurement tools of the eFilm workstation. The clarity of tumor border definition in all sequences and orientations (axial, sagittal, coronal) as well as the presence of artifacts was rated subjectively utilizing a 5-point scale, as previously described [15].

All images were obtained at the same anatomical level of the eye in order to depict relevant anatomical structures. The images were quantitatively evaluated utilizing the following metrics: (a) signal intensity (SI) values obtained from specific ROI measurements (mean, SD, and number of voxels) from the following 3 different anatomical eye structures: ciliary body, aqueous humor, vitreous humor, and (b) MR image background noise obtained from an artifact-free ROI. All ROIs were positioned on the same anatomical structures and were all of the same relative size (Fig. 1). All metrics based upon ROI measurements were performed utilizing an image postprocessing workstation (Nordic ICE software-NNL, software release).

The SNRs for the selected ROIs were calculated according to the following formula:

$$
S N R_{R O I}=\frac{S I_{R O I}}{1.53 \times S D_{B g}}, \quad S D_{S N R}=\frac{S D_{S I_{R O I}}}{1.53 \times S D_{B g}}
$$

$\mathrm{SI}_{\mathrm{ROI}}=$ mean signal intensity from the selected ROI,

$\mathrm{SD}\left(\mathrm{SI}_{\mathrm{ROI}}\right)=$ standard deviation of the mean signal intensity of the selected ROI,

$\mathrm{SD}_{\mathrm{Bg}}=$ standard deviation from the mean background ROI measurement.

The factor 1.53 is the Rayleigh noise distribution correction factor.

The CNRs (contrast-to-noise ratio) of the selected areas relative to the reference tissues were calculated according to the following formula:

$$
C N R=\frac{\left|S I_{R O I}-S I_{R E F}\right|}{1.53 \times S D_{B g}}, \quad S D_{S N R}=\sqrt{S D_{S N R}^{2}+S D_{R E F}^{2}}
$$

$\mathrm{SI}_{\mathrm{REF}}=$ mean signal intensity from the selected reference, $\mathrm{SD}_{\mathrm{REF}}=$ standard deviation from the mean reference.

Statistical analysis was performed using SPSS for Windows, software version 21 (SPSS, Chicago, IL, USA). Paired $t$ test was used to compare the means in order to determine statistical significant differences between SNR and CNR values in the different areas of the ciliary body. We considered $\alpha=0.05$.

Following the completion of MRI scanning, the experimental animal was humanely sacrificed, while still under anesthesia, with a $10-\mathrm{mL}$ air injection into the main ear vein. Subsequently, the right eye (tumor-bearing eye) was enucleated, fixed in $10 \%$ buffered formaldehyde solution (Formalde-Fresh, Fisher Chemical, Fairlawn, NJ, USA), and processed for cyanoacrylate sectioning. The sections were stained with hematoxylin-eosin and evaluated by light microscopy by an experienced examiner (V.H.).

\section{Results}

The histopathological examination of the enucleated eye confirmed the presence of intraocular melanoma (Fig. 2).

The T1-w and CISS-T2-w sequences revealed the anatomical regions of the ciliary body, the iris, and the lens (Fig. 3a, b) but CISS-T2-w images could not discriminate the tumor from the surrounding healthy tissue. The T1-w images showed a hyperintense area on the 12 o'clock position of the ciliary body, (Fig. 3a) which corresponded to the area of melanoma. 


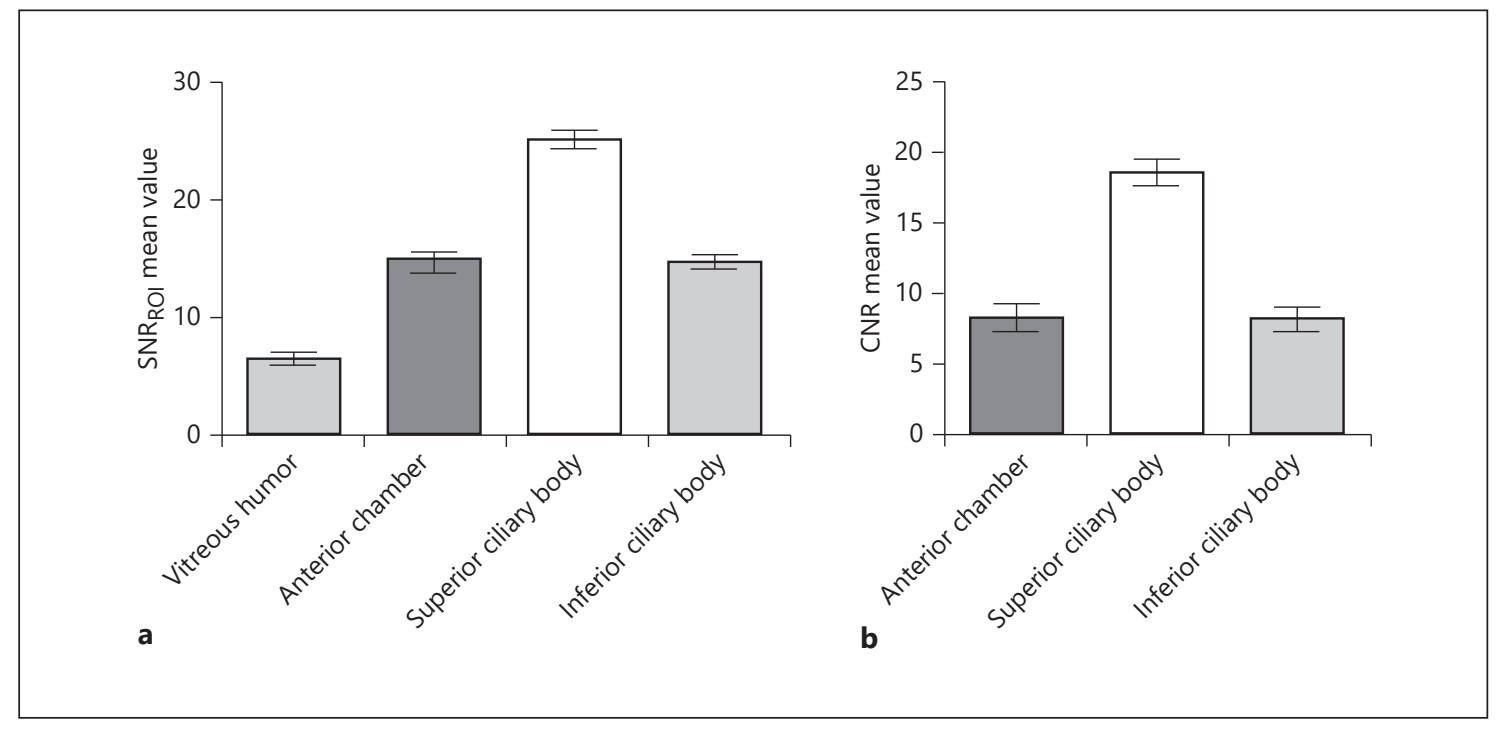

Fig. 4. a Graphical presentation of the signal-to-noise ratio (SNR) mean values and SD from 4 segments of the eye in the T1-w 3D-VIBE sequence. The mean SNR for the superior area of the ciliary body where the tumor was established displayed significantly higher value compared to the contralateral area. $\mathbf{b}$ Bar graph showing higher contrast-to-noise ratio (CNR) in the superior area of the ciliary body and thus a better discrimination in the targeted locus.

Table 2. Quantitative results of signal-to-noise ratio (SNR) and contrast-to-noise ratio (CNR) in the T1-w (3D-VIBE) sequence

\begin{tabular}{lrc}
\hline Areas & \multicolumn{1}{l}{ SNR } & $\begin{array}{c}\text { CNR (ref. } \\
\text { tissue: } V H)\end{array}$ \\
\hline Background & $28.1 \pm 15.1$ & - \\
Anterior chamber & $14.73 \pm 0.87$ & $8.27 \pm 1.03$ \\
Vitreous humor (VH) & $6.46 \pm 0.55$ & - \\
Superior area of the ciliary body & $25.54 \pm 0.73$ & $18.78 \pm 0.91$ \\
Inferior area of the ciliary body & $14.75 \pm 0.59$ & $8.29 \pm 0.81$ \\
\hline
\end{tabular}

The surface area occupied by the tumor measured $0.3595 \mathrm{~mm}^{2}$. The area occupied by the tumor was maxi$\mathrm{mal}$ in coronal sections $\left(0.5523 \mathrm{~mm}^{2}\right)$, followed by transverse $\left(0.3595 \mathrm{~mm}^{2}\right)$ and sagittal $\left(0.2958 \mathrm{~mm}^{2}\right)$ sections. The tumor area was associated with local retinal detachment and its proximity with the iris root resulted in poorly defined anterior borders.

The results of the quantitative analysis obtained from the T1-w (3D-VIBE) sequence are presented in Table 2. The tissue SNR values for the different anatomic structures as well as the CNR using the vitreous humor as reference are also reported in Figure 4. The mean SNR for the ciliary body in the 12 o'clock position (superior ciliary body) demonstrated a significant higher value (paired $t$ test, $t=112.34 ; p \approx 0.001)$ compared to the 6 o'clock position (inferior ciliary body). CNR was also found significantly higher in the superior area compared to the inferior area of the ciliary body (paired $t$ test, $t=86.1 ; p \approx$ $0.001)$.

\section{Discussion}

The present experimental study aimed at evaluating the power of surface-coil MRI to identify small peripheral intraocular melanomas. Ciliary body melanomas account for about $10 \%$ of all uveal melanomas and unlike posteriorly located tumors, their early detection is much more difficult. Slit-lamp biomicroscopy and indirect ophthalmoscopy are often inadequate to reveal a ciliary body mass or a mass located at the extreme choroidal periphery. Transscleral or transpupillary transillumination may provide some assistance in localization for pigmented lesions, but the presence of retinal detachment and ciliary band shadows can confuse interpretation. Ultrasound biomicroscopy may also be used to identify medium-sized tumors of the ciliary body or peripheral choroid, define their margins, demonstrate their internal echo, and guide biopsy, resection, or plaque radiotherapy [16]. 
MRI, in association with tools such as surface coils, gadolinium-diethylenetriamine penta-acetic acid (GdDTPA), and fat suppression has been extensively used in the detection and evaluation of intraocular lesions. Advantages include the multiplanar capabilities of MR imaging at high spatial resolution without the loss of spatial resolution that can occur with reconstructed computed images, the superior contrast resolution, and the lack of exposure to ionizing radiation [17].

In the present study, the fact that the optimal delineation of tumor margins was obtained in coronal sections may be associated with the anatomy of the rabbit orbit, in which the eyeball is rotated temporally by almost $90^{\circ}$. Accordingly, a coronal section in a rabbit orbit corresponds to a quasi-sagittal section for a human orbit and since tumor cell inoculation and subsequent development was aligned with the anterior-posterior axis along the superior peripheral fundus, a coronal section is more likely to delineate tumor borders. The fact that tumor borders were best identified in T1-weighted sections is in accordance with previous reports and may be associated with the histochemical characteristics of the lesions, including melanin concentration [18].

The semiquantitative analysis and the lack of contrastenhanced images may be considered weaknesses of the present study. On the other hand, the fact that the technique was capable of delineating small melanomas even without contrast enhancement supports the feasibility of the proposed methodology. However, the technique could be inappropriate for nonpigmented tumors, such as amelanotic melanomas which do not appear hyperintense in T1 sequences. Accordingly, future research in this area may include the administration of contrast-enhancing agents and the application of quantitative criteria in the analyses, such as the signal-to-noise ratio or contrast-to-noise ratio. However, results from this feasibility study imply that it may prove a valid tool in imaging of small peripheral choroidal lesions for both clinical and research purposes.

\section{Statement of Ethics}

All applicable international, national, and/or institutional guidelines for the care and use of animals were followed.

\section{Disclosure Statement}

The authors have no potential conflicts of interest relevant to this study to disclose.

\section{References}

1 Kernt M, Kampik A: Imaging of the peripheral retina. Oman J Ophthalmol 2013;6(suppl 1):S32-S35.

$\checkmark 2$ Witmer MT: Wide-field imaging of the retina. Kiss S. Surv Ophthalmol 2013;58:143-154.

-3 Shoughy SS, Arevalo JF, Kozak I: Update on wide- and ultra-widefield retinal imaging. Indian J Ophthalmol 2015;63:575-581.

4 Patel CK, Fung TH, Muqit MM, Mordant DJ, Brett J, Smith L, et al: Non-contact ultra-widefield imaging of retinopathy of prematurity using the Optos dual wavelength scanning laser ophthalmoscope. Eye (Lond) 2013;27: 589-596.

5 Reznicek L, Stumpf C, Seidensticker F, Kampik A, Neubauer AS, Kernt M: Role of wide-field autofluorescence imaging and scanning laser ophthalmoscopy in differentiation of choroidal pigmented lesions. Int J Ophthalmol 2014;7:697-703.

-6 Kernt M, Schaller UC, Stumpf C, Ulbig MW, Kampik A, Neubauer AS: Choroidal pigmented lesions imaged by ultra-wide-field scanning laser ophthalmoscopy with two laser wavelengths (Optomap). Clin Ophthalmol 2010;4:829-836.

Surface-Coil MRI for Small Choroidal Melanoma
7 Ghassemi F, Bazvand F, Hosseini SS: Pseudomelanoma at a referral center in Iran. J Ophthalmic Vis Res 2014;9:50-53.

$\checkmark 8$ Mithal KN, Thakkar HH, Tyagi MA, Bharwada RM, Billore PO: Role of echography in diagnostic dilemma in choroidal masses. Indian J Ophthalmol 2014;62:167-170.

$\checkmark 9$ Wycliffe ND, Mafee MF: Magnetic resonance imaging in ocular pathology. Top Magn Reson Imaging 1999; 10:384-400.

10 Kiratli H, Koç İ, Tarlan B: Orbital Extension of an Unsuspected Choroidal Melanoma Presumably through an Aqueous Tube Shunt. Ocul Oncol Pathol 2016;2:144-147.

11 Detorakis ET, Engstrom RE, Straatsma BR, Demer JL. Functional anatomy of the anophthalmic socket: insights from magnetic resonance imaging. Invest Ophthalmol Vis Sci 2003;44:4307-4313.

12 Hosten N, Bornfeld N, Wassmuth R, Lemke AJ, Sander B, Bechrakis NE, Felix R: Uveal melanoma: detection of extraocular growth with MR imaging and US. Radiology 1997; 202:61-67.

13 Blanco G, Saornil AM, Domingo E, Diebold Y, López R, Rábano G, Tutor CJ: Uveal melanoma model with metastasis in rabbits: effects of different doses of cyclosporine A. Curr Eye Res 2000;21:740-747.
14 Blanco PL, Marshall JC, Antecka E, Callejo SA, Souza Filho JP, Saraiva V, Burnier MN Jr: "Characterization of Ocular and Metastatic Uveal Melanoma in an Animal Model", IOVS, December 2005, Vol. 46, No. 12.

15 Tsiapa I, Tsilimbaris MK, Papadaki E, Bouziotis P, Pallikaris IG, Karantanas AH, Maris TG: High resolution MR eye protocol optimization: Comparison between 3D-CISS, 3DPSIF and 3D-VIBE sequences. Phys Med 2015;31:774-780.

16 Maberley DAL, Pavlin CJ, McGowan HD, Foster FS, Simpson ER: Ultrasound biomicroscopic imaging of the anterior aspect of peripheral choroidal melanomas. Am J Ophthalmol 1997;123:506-514.

17 Tien RD, Chu PK, Hesselink JR, Szumowski J: Intra - and paraorbital lesions: value of fat suppression MR imaging with paramagnetic contrast enhancement. AJNR 1991;12:245253.

18 Lemke AJ, Hosten N, Bornfeld N, Bechrakis NE, Frenzel D, Richter M, Felix R: Appearance of choroidal melanoma on high resolution MRI using $1.5 \mathrm{~T}$ with a dedicated surface coil in 200 consecutive patients. Rofo 1998; 169:471-478. 